\title{
Concentration, Wavelength and Temperature Dependent Refractive Index of Sugar Solutions and Methods of Determination Contents of Sugar in Soft Drink Beverages using Laser Lights
}

Belay A* $^{*}$ and Assefa $\mathbf{G}$

Applied Physics Program, School of Applied Natural Science, Adama Science and Technolgy University, Ethiopia

\begin{abstract}
The concentration, wavelength and temperature dependent refractive index of sugar solution has been investigated. The refractive index of sugar solutions (5\%-50\%) obtained using Red Diode Laser, Green Diode Laser and He-Ne Laser light were found to be in the range of (1.3357-1.4117), (1.3467-1.4272) and (1.3380-1.4140) respectively. The refractive index has linear relationship with the concentration of sugar solution. Mathematical expressions were also derived for the wavelength dependent refractive index of sugar solution by fitting Cauchy's equation to the experimental data using non-linear curve at the minimum discrepancy. The empirical expression of temperature dependent of refractive index of the sugar solution was determined; and the result indicates that, as the temperature increases the refractive index of the solutions decrease the method was also applied to determine the sugar contents in soft drink beverages (Pepsi, Coca, 7up, Sprite, Fanta and Mirinda). The results in $\mathrm{g} / 100 \mathrm{ml}$ are: (10.48 \pm 0.001$),(10.42 \pm 0.001),(10.42 \pm 0.001),(6.79 \pm 0.002),(7.03 \pm 0.001)$ and $(9.89 \pm 0.002)$ respectively. The techniques are simple, fast and inexpensive.
\end{abstract}

Keywords: Laser light; Refractive index; Temperature; Sugar solution and Soft drinks

\section{Introduction}

The refractive index $\mathrm{n}$ is a basic optical property of materials and its accurate value is often needed in many branches of physics and chemistry and has also several applications in many industries and materials to know the clarity of glasses and solid plastics [1-6]. By measuring the refractive index of a binary solution, one can determine the composition of the solution $[3,4]$. So, measurements of refractive index are widely used in many industrial and research applications to determine the concentration of the solution [3-6].

There are numerous methods for measuring the refractive index of a liquid solution that are well documented in literatures [1-10]. The diversity of methods is largely due to differences in applications, test objects and the required measurement accuracy. A separate class of devices for measuring the refractive index of transparent liquids consists of systems designed for examining liquids in cuvettes made of glass, quartz or other transparent materials. During the last decade, numerous practical devices based on the principle described above using various cuvettes have been constructed for determining the refractive index [6-9].

The refractive index varies with concentration, temperature, pressure, and wavelength [2-7,10,11]. Recent studies [2-4] provided the detailed study on the concentration mapping by the measurement of refractive index of liquids. Temperature coefficient of refractive index can also be used to calculate thermal expansion coefficient $[2,4]$. Several techniques are reported in literature for the measurement of concentration and temperature dependence of refractive index of sugar solution using various techniques [2-7]. Various techniques have been developed to determine the contents of sugar in solutions [12-14]. The techniques are Inter digital Capacitor Sensor to determine the contents of sugar in solution [12]; optical Coherence Tomography to determine blood glucose concentration in diabetic patients [13]; Quantification of sugar in soft drinks and fruit juices by Density, refractometry, infrared spectroscopy and statistical methods [14-16] are some of the techniques so far developed.
The above mentioned techniques are industrial made instrument used to determine the concentration of sugar in solution. Such kind of instrument is very expensive and also not available in most laboratories. So, in this research we used relatively inexpensive and locally constructed equilateral hallow prism and very sensitive laser light sources (Red and Green Diode, $\mathrm{He}-\mathrm{Ne}$ ) to determine the concentration, temperature and wavelength dependent refractive index of sugar solution and its contents in different soft drink beverages (Pepsi, Mirinda, Coca, Sprite, 7 up and Fanta) using Snell's law. An empirical expression was also derived for the wavelength dependent refractive index of sugar solution by fitting Cauchy's equation to the experimental data using non-linear curve at the minimum discrepancy.

\section{Materials and Methods}

\section{Materials}

Locally constructed equilateral hollow prism, Red and Green Diode laser, $\mathrm{He}-\mathrm{Ne}$ laser as light sources, digital electronic balance with a good accuracy for mass measurement were used. In addition, different apparatus such as measuring cylinder to measure the volume of water, mercury thermometer to control the temperatures of the solutions and magnetic stirrer with hot plate for heating and mixing the solution. In addition, deionized water as solvent, locally produced sugar and soft drink beverages (Pepsi, Coca, 7up, Sprite, Mirind and Fanta) were used as our sample.

*Corresponding author: Belay A, Applied Physics Program, School of Applied Natural Science, Adama Science and Technolgy University, P.O.Box 1888, Ethiopia, Tel: +251 22111 0400; E-mail: abebebelay96@gmail.com

Received June 14, 2018; Accepted June 27, 2018; Published July 04, 2018

Citation: Belay A, Assefa G (2018) Concentration, Wavelength and Temperature Dependent Refractive Index of Sugar Solutions and Methods of Determination Contents of Sugar in Soft Drink Beverages using Laser Lights. J Laser Opt Photonics 5: 187. doi: 10.4172/2469-410X.1000187

Copyright: ( 92018 Belay A, et al. This is an open-access article distributed unde the terms of the Creative Commons Attribution License, which permits unrestricted use, distribution, and reproduction in any medium, provided the original author and source are credited. 


\section{Methods of measuring concentration dependent refractive index of sugar solutions}

To measure the refractive index of different sugar solution standard samples of varying concentration of sugar $(\mathrm{m} / \mathrm{m}: 5 \%, 10 \%, 15 \%, 20 \%$, $25 \%, 30 \%, 35 \%, 40 \%, 45 \%, 50 \%$ ) were prepared by dissolving the sugar in deionized water. For example $5 \%$ of sugar solution means dissolving $5 \mathrm{~g}$ of sugar within $100 \mathrm{ml}$ of deionized water. Calibration curve developed from the graph of refractive index versus the concentration of the solution. Numerical procedure of fitting the experimental data was carried out by linear curve fitting using origin 8 software.

A theory and method for derivation of refractive index have been discussed elsewhere [1-2,5]. The angle of incidence is responsible for deviation produced in the path of light beam. The angle of deviation $\delta$ is minimum when incident and refracted angle of the prism are equal [5]. Therefore, the refractive index, $\mathrm{n}$ of the material filled inside the prism or solid prism of apex angle $(\alpha)$ can be calculated using eqn. (1) [7]

$$
n=\frac{\sin \left(\frac{\alpha+\delta}{2}\right)}{\sin \left(\frac{\alpha}{2}\right)}
$$

Figure 1 indicated the experimental setup of the measurements. A monochromatic source of light (Red, Green Diode laser and He-Ne laser) was allowed to fall at angle of incident on the face of the liquid prism and the angle of minimum deviation was determined for each laser light. During the measurement the solutions were filtered before pouring into the hollow prism. The hollow prism was also rinsed carefully after every measurement. Each measurement has taken three times.

\section{Wavelength dependent refractive Index of sugar solution}

An empirical relationship between the refractive index and wavelength of light for particular transparent materials can be expressed using Cauchy's equation eqn. (2) [17]

$$
n(\lambda)=A+\frac{B}{\lambda^{2}}+\frac{C}{\lambda^{4}}
$$

Where $\mathrm{n}$ is the wavelength dependent refractive index, $\lambda$ is the wavelength and A, B, C are the coefficients that can be determined for a materials. The fitting parameters are obtained by fitting Cauchy's equation to the experimental data by non-linear curve flitting using origin 8.6 software.

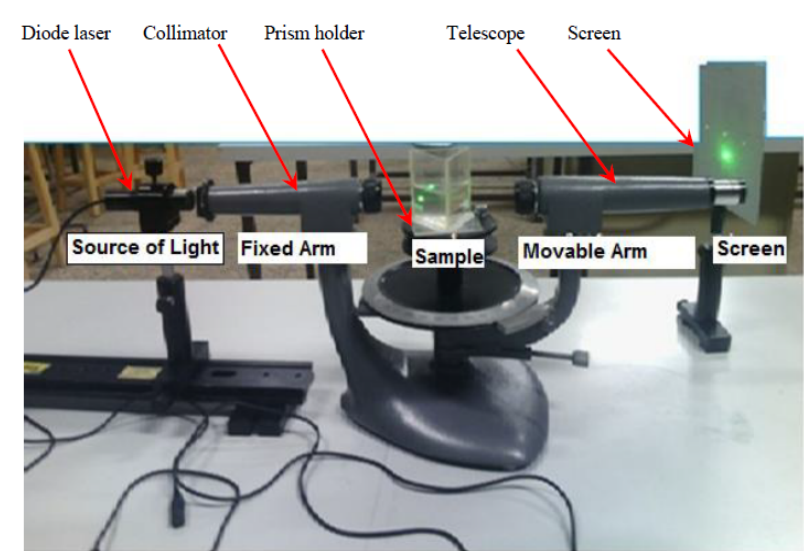

Figure 1: Experimental set up for the measurements of angle of minimum deviation using prism spectrometer.

\section{Methods of measuring temperature dependent refractive}

The temperature dependent refractive indices of the solution measured at the temperatures (308.15-343.15 K) inside hallow prism. The solutions were heated using magnetic stirrer with hot plate and its temperature controlled by placing the thermometer inside a solution. The angle of minimum deviation was measured at the interval of $5 \mathrm{~K}$ while the solution is cooling down from highest to lowest temperature. The temperature dependent refractive index measured for different mass fraction $(0 \%, 10 \%, 20 \% 30 \%, 40 \%$ and $50 \%)$. Calibration curve developed from the graph of refractive index versus temperature of the solution.

\section{Soft drink beverage solutions preparation}

In order to measure the sugar contents in soft drink beverages (Pepsi, Coca, 7up, Sprite, Fanta and Mirinda) each sample was poured into a beaker and stirred for 5-10 minutes by magnetic stirrer to remove gases within the samples. The refractive index of each solution was calculated from angle of minimum deviation using Equation (1). The sugar contents in soft drink beverages were determined using eqns. (2-4), which obtained from the graph of refractive index versus concentrations of sugar solution.

\section{Results and Discussion}

\section{Effect of concentration on the refractive index of the solutions}

Figure 2 shows the concentration dependent refractive indices of sugar solutions at room temperature measured using Red Diode Laser $(\lambda=650 \mathrm{~nm})$, Green Diode Laser $(\lambda=532 \mathrm{~nm})$ and He-Ne Laser $(\lambda=632.8 \mathrm{~nm})$ respectively. The refractive index of sugar solution (5$50 \%$ ) were found to be $1.3357-1.4117,1.3467-1.4272$ and 1.3380-1.4140 using Red Diode, Green Diode and $\mathrm{He}-\mathrm{Ne}$ laser light respectively. From experimental data we can find that RI of sugar solution increase linearly with the concentration. Eqns. (4) and (5) show calibration curve or refractive index versus sugar solutions obtained using Red Diode, $\mathrm{He}-\mathrm{Ne}$ and Green Diode laser respectively.

$$
\begin{aligned}
& n_{\text {Re dDiode }}=1.65 \times 10^{-3} c+1.3270 \\
& n_{H e-N e}=1.66 \times 10^{-3} c+1.3287 \\
& n_{\text {GreenDiode }}=1.77 \times 10^{-3} c+1.3382
\end{aligned}
$$

The slope of the linear curve fitting and standard errors are in the range of $(1.65-1.77) \times 10^{-3}$ and $(3.3840-1.375) \times 10^{-5}$ respectively. The

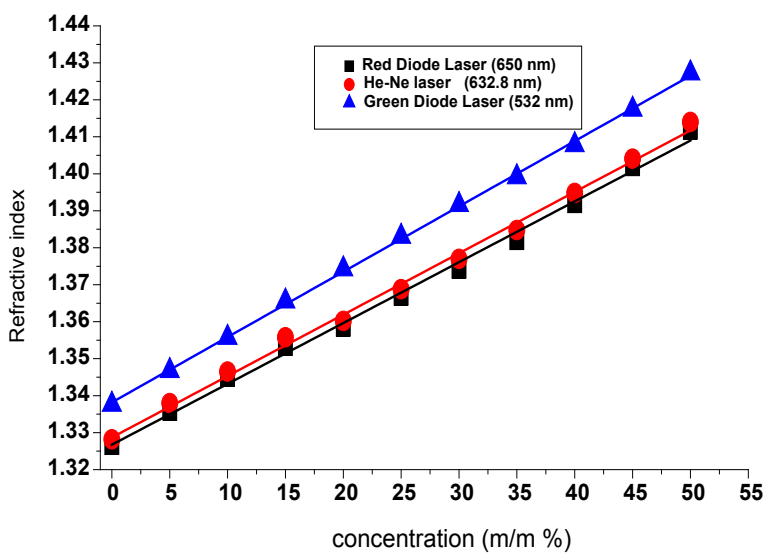

Figure 2: shows the refractive index versus concentrations of sugar solution using Red Diode, He-Ne and Green Diode laser in standard solutions. 
difference in the slope value indicated the dependence of refractive index on the wavelength of laser light.

A comparison of our experimental results with the available literature data indicated that the results are quite similar with those previously reported by other authors [2-4]. In Table the last two columns shows the refractive index of sucrose solutions reported using Abbe refractometer [4] and Sodium lamp at the wavelength $(589.29$ $\mathrm{nm})$ [2]. As indicated in Table 1 our data and the previously reported results using different techniques are quite consistent with deviation of the order of $\left(10^{-3}\right)$. Figure 3 displays the residual of the RI at different concentration of sugar solutions. It shows the good linear relationship between the measured RI and measured RI and concentration.

\section{Effects of wavelength on the refractive index}

Dispersion of light measures the change of the refractive index with wavelength. It can be explained by applying electromagnetic theory to the molecular structure of the materials. If an electromagnetic wave impinges on an atom or molecule the bound charge vibrate at the frequency of the incident wave. The bound charges have resonance frequency at certain wavelength. Figure 4 shows the refractive index versus wavelength of sugar solution (30\%) at room temperature. The dots are experimental data and solid lines are Cauchy's equation fitted to the experimental data. Over the visible wavelength regions, the Cauchy's equation quite fitted to the experimentally measured

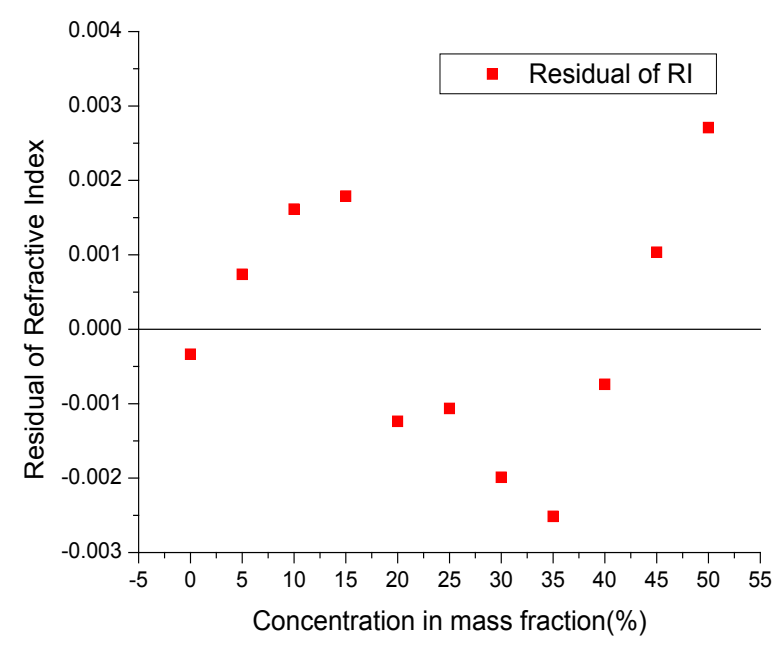

Figure 3: shows Residual of RI of sugar solution at room temperature. refractive indices. The three Cauchy fitting parameters extracted are: $\mathrm{A}=1.38399, \mathrm{~B}=0.01611 \mu \mathrm{m}^{2}, \mathrm{C}=0.00516 \mu \mathrm{m}^{4}$ and the correlation coefficient $\left(\mathrm{R}^{2=} 0.97\right)$ respectively. The obtained fitting parameters are similar with the previously reported refractive indices of liquid crystals [18]. The three Cauchy coefficients are the peculiar properties of the materials.

The empirical expression for the wavelength dependent refractive index of sugar solution (30\%) obtained by fitting Cauchy's equation to the experimental data using non-linear curve are given by the following Equation (7)

$$
n(\lambda)=1.3839-\frac{16.11 \times 10^{-3}}{\lambda^{2}}+\frac{5.16 \times 10^{-3}}{\lambda^{4}}
$$

Using eqn. (6), the calculated refractive index at the wavelength of 532 and $650 \mathrm{~nm}$ are 1.3915 and 1.3748 respectively.

\section{Effects of temperature on refractive index of sugar solutions}

Figure 5 illustrates the dependence of refractive index on temperatures investigated using laser lights with different sugar concentrations. It indicates that at the fixed concentration, the refractive index decrease linearly with the temperatures (308.15-340.15 K). From the fitting curve the slope increase as the concentration of the solution increases. The linear equations relate the refractive index with the temperature for $40 \%$ sugar solution can be expressed using eqns. (8) and (9).

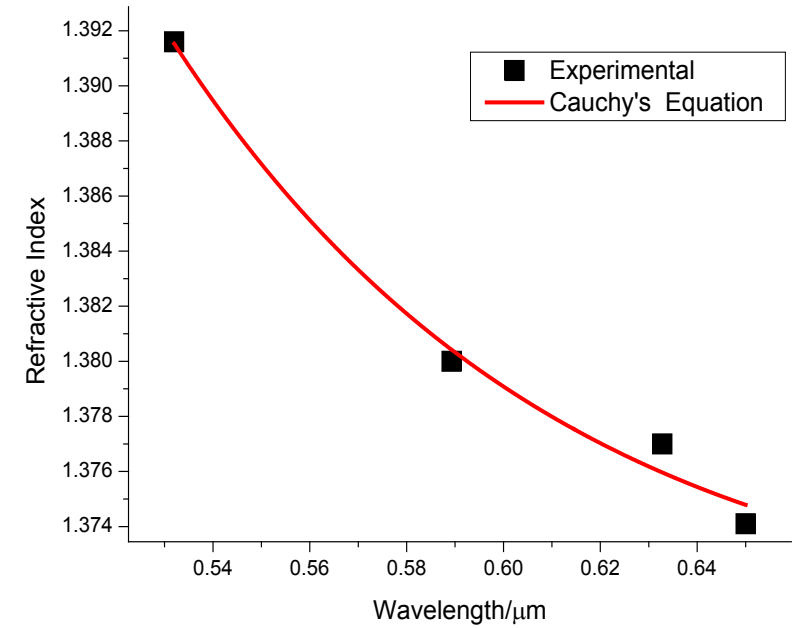

Figure 4: Wavelength dependent refractive index for sugar solution of $30 \%$ at room temperature $\mathbf{a}$. the dot indicate experimental results and $\mathbf{b}$. the line indicate Cauchy's Equation.

\begin{tabular}{|c|c|c|c|c|}
\hline Concentration (\%) & $\begin{array}{c}\text { RI values Using Red } \\
\text { Diode }\end{array}$ & RI values Using He-Ne & $\begin{array}{c}\text { RI values Using Green } \\
\text { Diode }\end{array}$ & Literatures value [4] \\
\hline 0 & 1.3264 & 1.3282 & 1.3376 & 1.3360 \\
\hline 5 & 1.3357 & 1.3380 & 1.3467 & 1.3412 \\
\hline 10 & 1.3448 & 1.3465 & 1.3557 & 1.3481 \\
\hline 15 & 1.3532 & 1.3558 & 1.3655 & 1.3553 \\
\hline 20 & 1.3584 & 1.3602 & 1.3742 & 1.3623 \\
\hline 30 & 1.3668 & 1.3688 & 1.3830 & 1.3775 \\
\hline 35 & 1.3741 & 1.3770 & 1.3916 & \\
\hline 45 & 1.3818 & 1.3848 & 1.3991 & \\
\hline 50 & 1.3918 & 1.3948 & 1.4078 & \\
\hline
\end{tabular}

Table 1: Comparison of refractive index of sugar solutions of our results using different laser light $(532,632.8$ and $650 \mathrm{~nm})$ and the reported data using sodium lamp ( 589.29 $\mathrm{nm}$ ) and Abbe refractometer. 

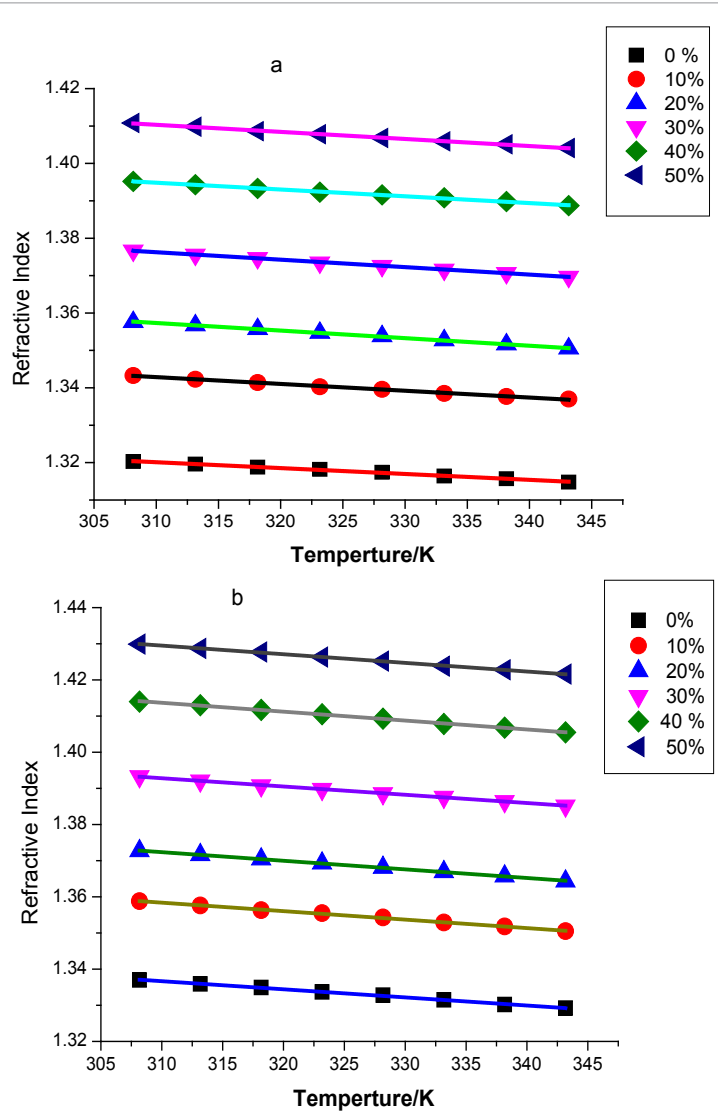

Figure 5: The dependence of refractive index of sugar solution on temperature at different concentrations using a. Red diode laser $(650 \mathrm{~nm})$ and b. Green Diode laser $(532 \mathrm{~nm})$.

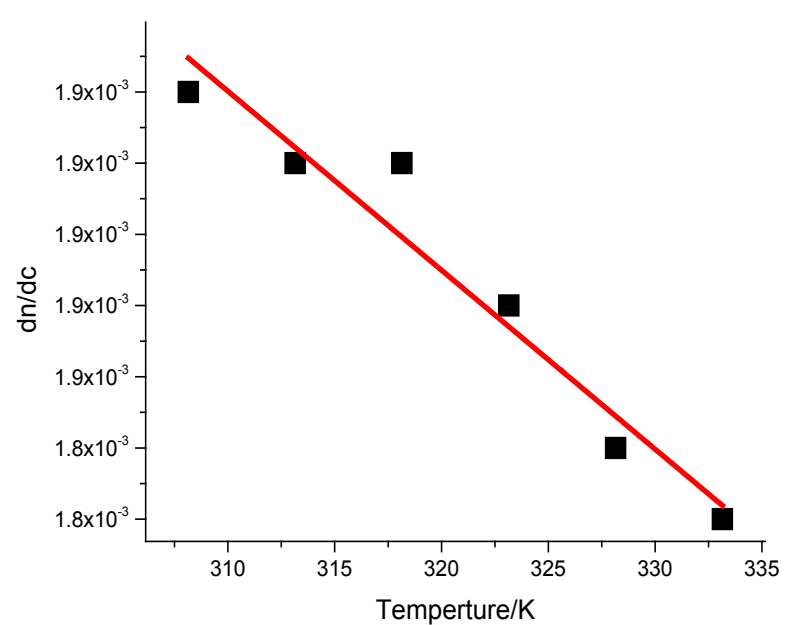

Figure 6: Shows the variation of $\mathrm{dn} / \mathrm{dc}$ vs temperature.

$$
\begin{aligned}
& n_{\text {RedDiode }}=-1.81 \times 10^{-4} T+1.40 \\
& n_{\text {GreenDiode }}=-2.46 \times 10^{-4} T+1.42
\end{aligned}
$$

Where, $\mathrm{n}_{\text {RedDiode }}$ and $\mathrm{n}_{\text {GreenDiode }}$ represents the refractive index obtained using Red and Green Diode laser respectively. The coefficients of the temperature deduced from the experimental data are the order of $10^{-4}$ for all lasers light. The temperature coefficient of RI for different concentrations can be used to calculate the thermal expansion coefficient [6].

\section{Variation of $\mathrm{dn} / \mathrm{dc}$ with temperature}

Figure 6 shows the variation of $\mathrm{dn} / \mathrm{dc}$ vs. temperature. We can see that the derivative of refractive index with respect to concentration change highly with temperature and the depending coefficients are in the order of $10^{-7}$. The $\mathrm{dn} / \mathrm{dc}$ of the solution decreases with the increases of the temperature. From fitting linear equation to experimental data the following empirical eqn. (9) obtained

$$
\frac{d n}{d c}=5.028 \times 10^{-7} T+0.00201
$$

\section{Determination of sugar contents in soft drink beverages solutions}

Equations (4-6) show the relation between the refractive index and concentration of sugar solutions using three lasers light. Using eqn. (4) the contents of sugar in soft drink beverages solutions (Pepsi, Coca, $7 \mathrm{Up}$, Sprite, Fanta and Mirinda) were determined. In Table 2 the first column shows the average refractive index in different soft drinks measured using Red Diode laser $(\lambda=650 \mathrm{~nm})$.

The values of sugar contents determined in soft drinks using this technique is within the range of $8.62-12.30 \%$ which is also quite similar with previously reported by other techniques such as densitometer and refractometer, Spectrophotometeric, and HPLC [19-21]. The assessment of the sugar level of the ten soft drinks samples studied using densitometer and refractometer is within the range of $7-14 \%$ [19]. Also the sugar contents of soft drinks available in Nigeria studied by spectrophotometer are in the range of 9.91-13.55\% [20]. The sugar contents of the three soft drinks (Pepsi, Coca-cola and Sprite) studied using powerful instruments HPLC are 11.40, 11.10 and 8.20 respectively [21]. From the study results the new technique analyzed the level of sugar within the acceptable limit of regulatory standard. Moreover, the techniques are also simple, fast and inexpensive.

\section{Conclusions}

We have investigated the concentration, temperature and wavelength dependent refractive index of sugar solution and an empirical expression established for the concentration and temperature coefficients of the solutions. By this method the contents of sugar in soft drink beverages determined and the results are quite similar with the previously reported literatures. These findings can help to improve the fundamental understanding of binary mixtures and significant for the determinations of the concentration. The techniques are also simple, fast and inexpensive.

\begin{tabular}{|c|c|c|c|}
\hline Items & Refractive index & Sugar contents (\%) or $\mathbf{g} / \mathbf{1 0 0} \mathbf{~ m l}$ & Content of sugar soft drink beverages from other literatures \\
\hline Pepsi & 1.3473 & $12.30 \pm 0.001$ & $7-14[19]$ \\
\hline Coca & 1.3472 & $12.24 \pm 0.001$ & $9.91-13.55[20]$. \\
\hline 7 U & 1.3472 & $12.24 \pm 0.002$ \\
\hline Sprite & 1.3412 & $8.62 \pm 0.001$ & \\
\hline Fanta & 1.3416 & $8.85 \pm 0.001$ \\
\hline Mirinda & 1.3463 & $11.70 \pm 0.002$ \\
\hline
\end{tabular}

Table 2: The Refractive indices and sugar contents of soft drink beverages (Coca, Pepsi, Mirind, sprite, 7up and Fanta) and literatures report. 
Citation: Belay A, Assefa G (2018) Concentration, Wavelength and Temperature Dependent Refractive Index of Sugar Solutions and Methods of Determination Contents of Sugar in Soft Drink Beverages using Laser Lights. J Laser Opt Photonics 5: 187. doi: 10.4172/2469-410X.1000187

Page 5 of 5

\section{References}

1. Huang YX, Chinese Journal of Medical Physics, 11: 21-26 (1994).

2. Subedi DP, Panta GP (2006) Electrical characterization of aluminum (Al) thin films measured by using four- point probe method. Kathmandu University Journal of Science, Engineering and Technology 8: 31-36.

3. Singh VK, Jaswal BBS, Kumar V, Prakash R, Rai P (2013) Application of HeNe Laser to Study of the Variation of Refractive Index of Liquid Solutions with the Concentration. J Integrated Sci Tech 1: 13-18.

4. Tan CY, Huang YX (2015) Dependence of Refractive Index on Concentration and Temperature in Electrolyte Solution, Polar Solution, Nonpolar Solution, and Protein Solution. J Chem Eng 60: 2827-2833.

5. Yunus WMBM, Rahman ABA (1988) Refractive index of solutions at high concentrations. Appl Opt 27: 3341-3343.

6. Shao D, Tian L, Chen J, and Chen X (2010) Improved retroreflection method for measuring the refractive index of liquids. Appl Opt 49: 3049-3052.

7. Grange BW, Stevenson WH, Viskanta R (1976) Refractive index of liquid solutions at low temperatures: an accurate measurement. Appl Opt 15: 858859.

8. Vilitis O, Shipkovs P, Merkulov D (2009) Determining the refractive index of liquids using a cylindrical cuvette. Meas Sci Technol 20: 117001.

9. Narayanan VA, Narayanan R, J Chem Educ 74221(1197).

10. Jenkin DD (1982) Refractive indices of solutions. Phys Educ 17: 82-83.

11. Bashkatov AN, Genina EA (2003) Water refractive index in dependence on temperature and wavelength: a simple approximation. Proc. SPIE 5068, Saratov Fall Meeting 2002: Optical Technologies in Biophysics and Medicine IV.
12. Angkawisittpan N, Manasri T (2012) Determination of Sugar Content in Sugar Solutions using Interdigital Capacitor Sensor. Meas Sci Rev 12: 8-13.

13. Esenaliev RO, Larin KV, Larina IV (2001) Noninvasive monitoring of glucose concentration with optical coherence tomography. Opt Lett 26: 992-993.

14. Ponnadurai R, Sabina JL, Philipe R, Frédéric C, Heena S et al. (2004) Quantification of Sugars in Soft Drinks and Fruit Juices by Density, Refractometry, Infrared Spectroscopy and Statistical Methods. S Afr J Chem 57: 24-27.

15. Henderson SK, Carol AF, Domijan JD (1998) Determination of Sugar Content in Commercial Beverages by Density: A Novel Experiment for General Chemistry Courses. J Chem Educ 75: 1122-1123.

16. Cadet F, Bertrand D, Robert P, Maillot J, Dieudonne J (1991) Quantitative Determination of Sugar Cane Sucrose by Multidimensional Statistical Analysis of their Mid-Infrared Attenuated Total Reflectance Spectra. Appl. Spectrosc. 45: 166-172.

17. Jenkins FA and White HE (1981) Fundamentals of Optics ( $3^{\text {rd }}$ edn.), McGrawHill 1957.

18. Li J, ST Wu (2004) Extended Cauchy equations for the refractive indices of liquid crystals. J Appl Phys 95: 896-901.

19. Agbazue VE, Ibezim A, Ekere NR (2014) Assessment of sugar levels in different soft drinks. Int. J. Chem. Sci.12: 327-334.

20. Sodamade A (2014) International Journal of Science and Research (IJSR) 3 567-569.

21. Ventura EE, Davis JN, Goran MI (2010) Sugar content of popular sweetened beverages based on objective laboratory analysis: focus on fructose content. Obesity (Silver Spring) 